\title{
LHCb inputs to astroparticle physics
}

\author{
Marianna Fontana* \\ INFN Cagliari and CERN \\ E-mail: marianna.fontana@cern.ch
}

The LHCb experiment has the unique possibility, among the LHC experiments, to be operated in fixed target mode, using its internal gas target SMOG. The excellent detector capabilities for vertexing, tracking and particle identification allow to measure exclusive particle production for collisions of protons on different nuclei at an energy scale of $\sqrt{s}_{N N} \sim 100 \mathrm{GeV}$, providing valuable inputs to the modelling of cosmic ray interactions in the atmosphere and in the cosmos. In particular, by operating SMOG with helium, LHCb performed the first measurement of antiproton production in proton-helium collisions. The results improve the accuracy of the prediction for secondary antiproton production in cosmic rays at the energy scale accessible to space-borne detectors.

EPS-HEP 2017, European Physical Society conference on High Energy Physics 5-12 July 2017

Venice, Italy

${ }^{*}$ Speaker. 


\section{Introduction}

Precision measurements of the cosmic ray antiproton fraction provide a sensitive indirect probe for Dark Matter. In the last years the PAMELA [1] and AMS-02 [2] experiments reported an important improvement in the experimental determination of the $\bar{p} / p$. The uncertainty on the measurements are dominated, particularly in the $10-100 \mathrm{GeV}$ range, by the limited knowledge of the $\bar{p}$ production cross-section in the $p \mathrm{He}$ scattering. LHCb performed the first measurement of antiproton production from collisions of LHC proton beams on He nuclei at rest, thanks to its gas injection system.

The LHCb detector [3] is a single-arm forward spectrometer covering the pseudorapidity range $2<\eta<5$, designed for the study of particles containing $b$ or $c$ quarks. The detector is equipped for an excellent vertexing, tracking and particle identification (PID). These features allow the detector to be used also as a fixed-target experiment. The SMOG (System for Measuring Overlap with Gas) [4] device provides the target through the injection of a small amount of noble gas ( $\mathrm{He}, \mathrm{Ne}$, $\mathrm{Ar}$ ) inside the primary vacuum around the LHCb vertex locator (VELO). The design gas pressure $\left(2 \times 10^{-7} \mathrm{mbar}\right)$ is small enough not to perturb the LHC operation.

\section{Antiproton production in $p$ He collisions}

The analysis is performed on data taken during May 2016, operating the SMOG target with helium gas. A proton beam with an energy of $6.5 \mathrm{TeV}$ is used, which corresponds to a nucleonnucleon centre-of-mass energy $\sqrt{s}_{N N}=110.5 \mathrm{GeV}$. The data recorded for the measurement are taken when a bunch in the beam pointing toward the $\mathrm{LHCb}$ detector crosses the detector without a corresponding bunch in the other beam. The online event selection is performed by a trigger, which consists of a hardware stage (L0), followed by a two-steps software stage (HLT1-HLT2), which applies a full event reconstruction. In both steps the requirement for the analysis are very loose. The hardware stage is triggered by any activity in the SPD detector, while in the software stage is required the presence of at least one reconstructed track in the VELO.

The signal $\bar{p}$ are the ones produced directly in $p \mathrm{He}$ collisions from resonance decays. The $\bar{p}$ produced in the hyperon decays are instead considered as a background and subtracted from the result. Another source of background is the LHC residual vacuum. Its typical level $\left(10^{-9} \mathrm{mbar}\right)$ is not negligible with respect to the injected gas pressure. To precisely account for this background, part of the data was acquired before the injection of the helium gas (no-gas data). The analysis indicates that the contamination is at the level of $1 \%$. The multiplicity distribution confirms that most of the gas is hydrogen, as seen in the rest gas analysis on the static vacuum.

The best reconstruction efficiency is obtained in the fiducial region $-700<z<100 \mathrm{~mm}$, along the direction of the beam. The measurement is performed in two dimensional kinematic bins in momentum $(p)$ and transverse momentum $\left(p_{T}\right)$ in the range $12<p<110 \mathrm{GeV}, 0.4<$ $p_{T}<4 \mathrm{GeV}$. These limits correspond to the acceptance of the two RICH detectors providing the proton identification. Only tracks reconstructed with high quality are selected. Requirements on the impact parameter quantity are applied in order to select only tracks produced at the primary vertex. The reconstruction efficiency, which includes the tracking efficiency and acceptance effects of the detector is determined from simulation samples. A three dimensional binning in the $p, p_{T}$ 
and $z$ variables is chosen. The tracking efficiency determined from simulation is corrected for simulation/real data differences, using $p p$ calibration samples.

Among the selected sample of negatively charged tracks, the identification of $\bar{p}$ is based on the response of the RICH detectors. Two variables, representing the difference of the log likelihood between the proton and pion $\left(D L L_{p-\pi}\right)$ and proton and kaon $\left(D L L_{p-K}\right)$ hypothesis are built to discriminate the different particle species. The number of signal events is determined with a twodimensional binned maximum likelihood fit. Three sets of templates are obtained from simulation predictions and calibration samples in the $p \mathrm{He}$ and $p p$ data for the different particle species. The fit procedure is illustrated in Fig. 1.
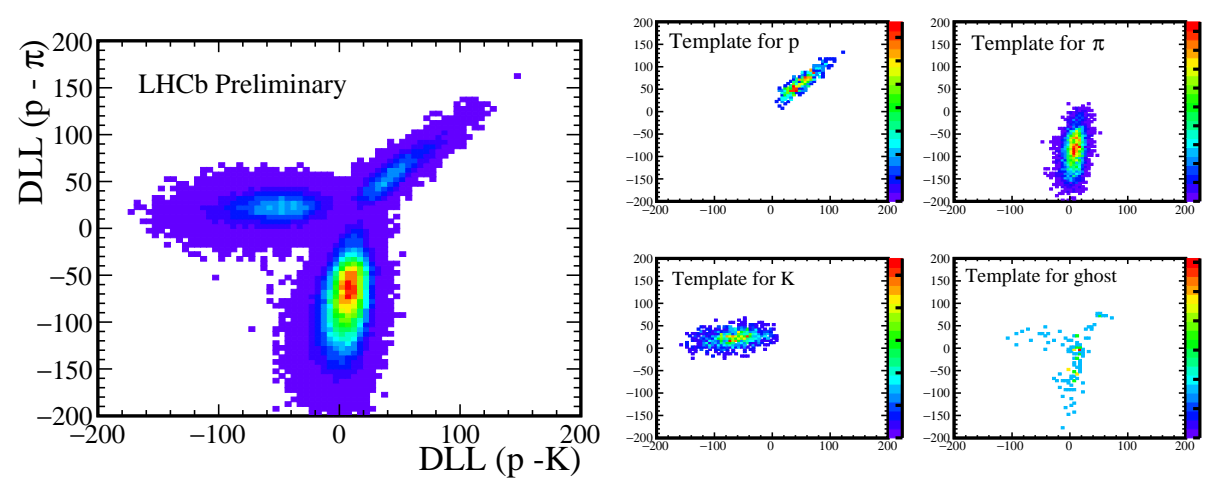

Figure 1: Example of two-dimensional template fit to the DLL distributions. On the left the two-dimensional distribution for data and on the right the templates for the four categories.

The $\bar{p}$ fraction in each (p, pT) bin is estimated from the average result of the fit performed with the different templates used for a given bin. The yield of $\bar{p}$ is dominated by systematic uncertainty, coming for most bins from the error on the $\bar{p}$ fraction from the PID analysis, or by the uncertainty on the tracking efficiency. Large relative uncertainties, up to $26 \%$, affect the bins at the borders of the detector acceptance and, for the intermediate momentum region, in the transition region between the two RICH detectors. For the other regions, the accuracy is typically a few per cent.

The SMOG device does not allow a precise measurement of the gas pressure inside the beam pipe. Instead, the elastic proton scattering on He atomic electrons has a very well known crosssection and hence is used as a normalisation process. Electrons are selected through track-quality requirements, electron identification in the electromagnetic calorimeter and by vetoing other activity in the detector. The criteria are very loose, with high efficiency. The luminosity is determined from the background-subtracted yield of scattered electrons $N_{e}$, the known cross-section $\sigma_{p e^{-}}$and the electron reconstruction efficiency $\varepsilon_{e}$, as $\mathscr{L}=N_{e} /\left(Z_{H e} \times \sigma_{p e^{-}} \times \varepsilon_{e}\right)$, where $Z_{H e}=2$ is the helium atomic number.

The double differential $\bar{p}$ production cross-section is determined for each bin in the $\left(p, p_{T}\right)$ plane. The raw yields in each bin are corrected for the various efficiencies. The procedure is validated on simulation, by performing the full analysis. No evidence for a distortion of the simulated cross-sections was found in the validation. The results [5] are shown in Fig. 2, where they are 
compared to the EPOS-LHC prediction [6]. The double differential shape is found to be in good agreement with the model prediction, while the absolute production rate is larger by about a factor 1.5. The total inelastic cross-section is also determined from the measured total yield of recorded collisions within the fiducial region. The result is

$$
\sigma_{\text {inel }}^{L H C b}(p H e), \sqrt{s_{N N}}=110 \mathrm{GeV}=(140 \pm 10) \mathrm{mb}
$$

to be compared with the EPOS LHC prediction

$$
\sigma_{\text {inel }}^{\text {EPOSL-HC }}(p H e), \sqrt{s_{N N}}=110 \mathrm{GeV}=118 \mathrm{mb}
$$

The ratio between the two quantities is $1.19 \pm 0.08$, which implies a larger $\bar{p}$ multiplicity per inelastic collision with respect to EPOS-LHC.

In Fig. 2 the data are also compared with three other models implemented in CRMC: EPOS1.99 [7], HIJING 1.38 [8] and QGSJet-II-04 [9]. The pre-LHC version of EPOS gives a better prediction of the multiplicity. HIJING predicts a lower inelastic cross-section (100 mb), while reproducing well the measured absolute $\bar{p}$ production cross-section values.

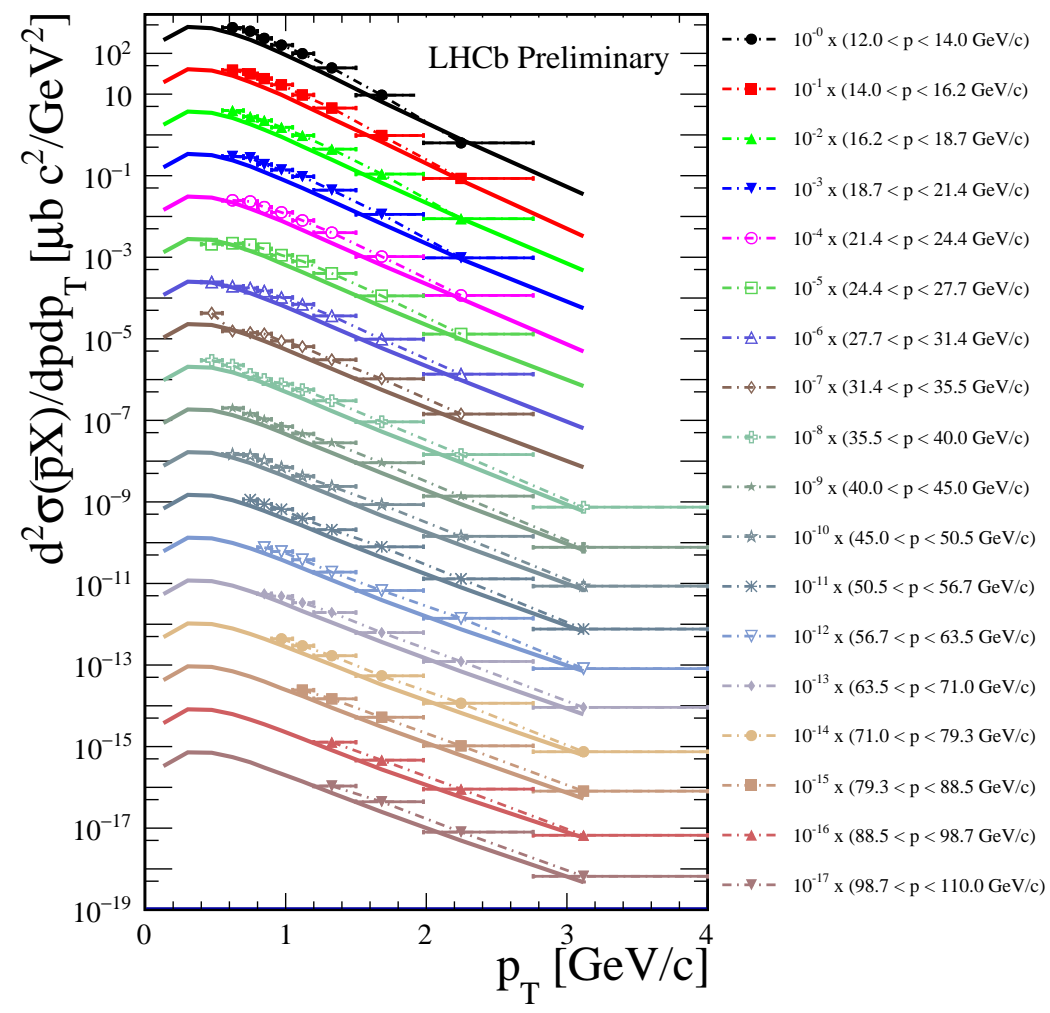

Figure 2: Result for the $\bar{p}$ cross-section measurement. The data points show the double differential crosssection as a function of $\mathrm{pT}$ in the 18 momentum bins, with values successively scaled by a factor 0.1 to improve the readibility of the plot. The solid curves show the EPOS LHC absolute predictions, scaled with the same factors as the data. The errors bars, barely visible, show the uncorrelated uncertainty only. 


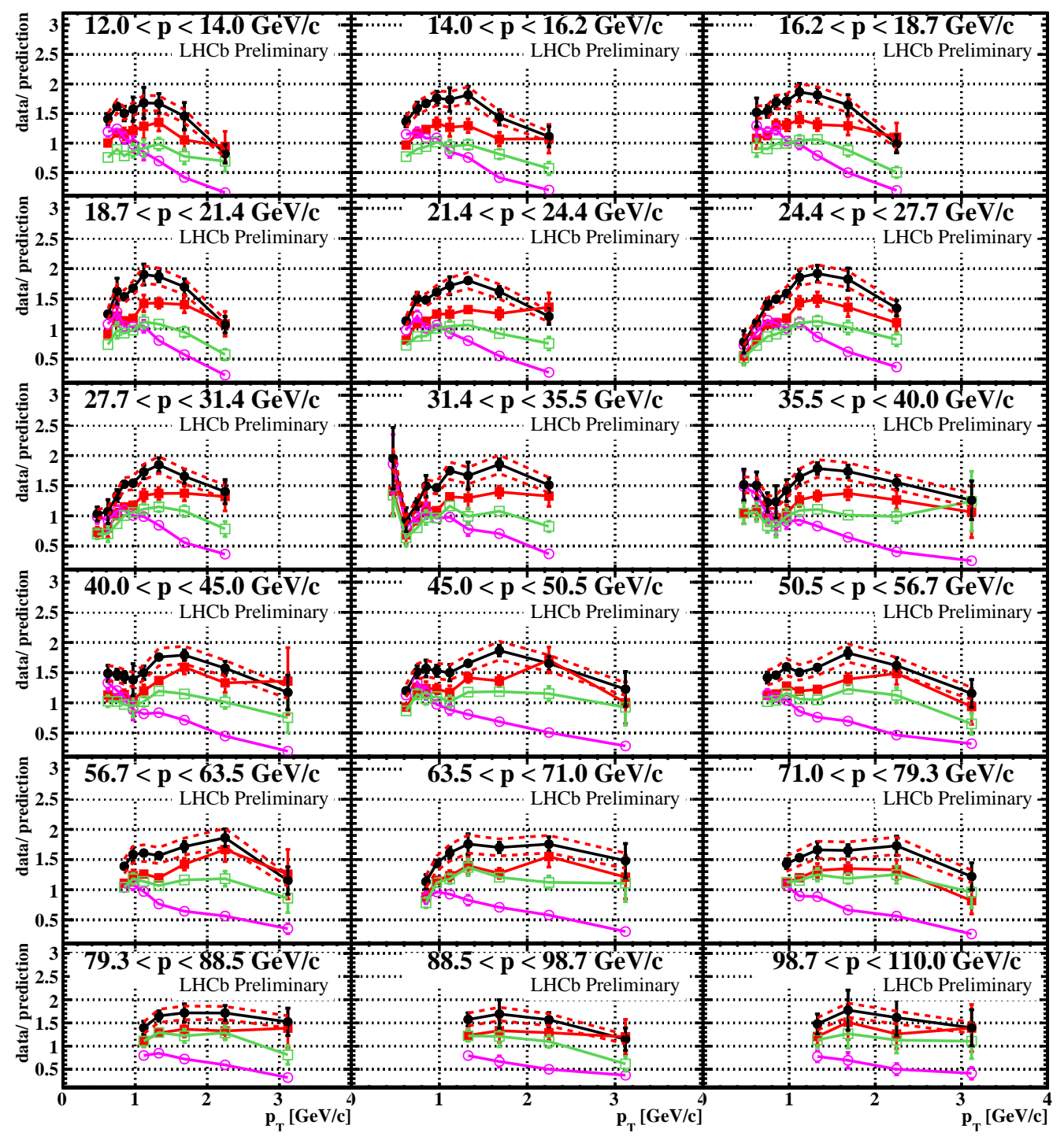

Figure 3: Result for the $\bar{p}$ double differential cross-section measurement as a function of $p_{T}$ in the 18 momentum bins, compared to absolute predictions from different models. The plots show the ratio of data over simulation for (black round closed symbols) EPOS LHC, (red squared closed symbols) EPOS 1.99, (green squared open symbols) HIJING 1.38, and (violet round open symbols) QGSJETII-04. The values of the data points are successively scaled by a factor 0.1 to improve the readibility of the plot.

This result is expected to have a sizeable impact on the accuracy of the prediction for the $\bar{p} / p$ ratio expected in cosmic rays from spallation of primary protons on the interstellar medium. Further studies are foreseen exploiting the data collected at $\sqrt{s_{N N}}=86.6 \mathrm{GeV}$ during November 2016, and the measurement of the contribution due to secondary hyperon decays.

\section{References}

[1] PAMELA collaboration, O. Adriani et al., JETP Letters 96 (2013) 621

[2] AMS collaboration, M. Aguilar et al., Phys. Rev. Lett. 117 (2016) 091103 
[3] LHCb collaboration, A. A. Alves Jr. et al, JINST 3 (2008) S08005

[4] LHCb collaboration, R. Aaij et al, JINST 9 (2014) P12005

[5] LHCb collaboration, R. Aaij et al, CERN-LHCb-CONF-2017-002

[6] T. Pierog et al., Phys. Rev. C92 (2015) 034906

[7] T. Pierog and K. Werner, Nucl. Phys. Proc. Suppl. 196 (2009) 102

[8] M. Gyulassy, Comput. Phys. Commun. 83 (1994) 307

[9] S. Ostapchenko, Phys. Rev. D83 (2011) 014018 